\title{
SURFACE LITHIUM AND COOL SPOTS
}

\author{
F. C. FEKEL \\ Center for Automated Space Science and \\ Center of Excellence in Information Systems \\ Tennessee State University, \\ Nashville, TN 37203, USA
}

\begin{abstract}
Observations of lithium in sunspots resulted in the prediction of significant lithium equivalent-width variability in chromospherically active stars. Lithium observations of several types of active stars, such as pre-main-sequence stars and late-type dwarfs in very young clusters, whose members are assumed to be nearly coeval, as well as post-main-sequence objects, show a significant range of abundances. Thus, a number of researchers over the past decade have investigated star spots as the possible cause of the various observed lithium-abundance spreads. Observationally, some evidence has been found for lithium equivalent-width variations. Computed synthetic spectra indicate that under certain conditions significant variations should be detected, but such conditions are not often met except perhaps in the most active stars. While variations have been claimed for some $\mathrm{T}$ Tauri stars, simultaneous photometry and spectroscopy will be needed to explore the complex relation of spots and lithium-abundance variations. The fact that some post-main-sequence stars have lithium abundances similar to those of pre-main-sequence stars complicates the identification of isolated pre-main-sequence systems.
\end{abstract}

\section{Patient history}

In his seminal work Herbig (1965) surveyed the lithium abundances of about $100 \mathrm{~F}$ and $\mathrm{G}$ dwarfs. He found a statistical correlation between $\mathrm{Ca}$ II emission and lithium abundance that suggested that the lithium abundance in a star decreases with age. Skumanich (1972) provided a quantitative relation for Ca II emission decay with age and found that the relation for rotational 
spin-down has a similar form, while the relationship for lithium depletion was somewhat different. Nevertheless, from the initial studies the expectation arose that the abundance of lithium might well provide an additional age calibrator for late-type dwarfs. The convection zone in late-type stars deepens with decreasing mass, providing increased mixing of lithium to the hot interior of the star where it is destroyed and making the lithium depletion rate mass dependent. Duncan (1981) examined the Ca II emission and lithium abundances of over 100 field F5-G5 dwarfs and subgiants. He confirmed that in a statistical sense lithium decreases as a main-sequence star ages. However, he also found a number of anomalous solar-type stars with low levels of chromospheric activity but large lithium abundances. Another problem with lithium surfaced when Duncan and Jones (1983) found a large spread in the lithium abundances of low-mass Pleiades stars, later confirmed by Soderblom et al. (1993). Such a spread in presumably coeval stars of the same mass argues that at least one additional parameter significantly affects the depletion rate in Population I dwarfs.

\section{Predicted disease}

By far the most easily measured lithium line is that of the resonance doublet at $6707.8 \AA$. Since the ionization potential is low, the equivalent width of this feature is particularly sensitive to temperature and the line weakens due to ionization as the temperature increases. For a given equivalent width the derived abundance changes in the same sense as the assumed effective temperature. For example, at solar temperatures the log lithium abundance changes by about 0.08 per $100 \mathrm{~K}$ (Pasquini et al. 1994). Abundance changes are even more sensitive at lower temperatures. At $4000 \mathrm{~K}$ an increase of $100 \mathrm{~K}$ in the assumed photospheric temperature increases the log lithium abundance by about 0.25 (Pilachowski et al. 1990).

Because of this sensitivity, it should not be surprising that the lithium line strength is greatly enhanced in cool sunspots relative to the Sun's quiet photosphere (Traub and Roesler 1971, Giampapa 1984). Giampapa (1984) applied those results to chromospherically active stars, which have extensive non-uniform spot distributions. He predicted that such stars would have significant variations in the strength of the lithium line as the spots rotated in and out of view. Since typically only one measurement of the lithium abundance is obtained per star, the observed lithium equivalent widths and lithium abundances of chromospherically active stars would produce significant scatter in correlations with other data, an unrecognized prescription for disaster. 


\section{Health tests}

An early attempt to examine the reality of this prediction was undertaken by Robinson et al. (1986). They obtained spectra of three broad-lined active stars and searched for lithium equivalent-width changes correlated with the stars' rotation periods. They found possible variations of about $20 \%$ in two of the stars, PZ Tel and AB Dor. However, they noted that measurements of the control line, Ca I $6717.7 \AA$, whose changes should have been in phase with those of lithium, did not support the conclusion. In addition, they were unable to carry out simultaneous photometric observations.

A similar spectroscopic test was made by Boesgaard (1991), who observed six active dwarfs with spectral types from F6 to G5, including $\chi^{1}$ Ori and 111 Tau. The stars were chosen because they all had rotational periods determined from Ca II K line modulation. Boesgaard (1991) measured both the lithium line and the nearby Fe I line at $6705.1 \AA$. She found that the lithium equivalent widths had no variations greater than $1 \%$ and thus, concluded that her patient was healthy.

Strom et al. (1989) estimated lithium abundances for a large sample of $\mathrm{K}$ and $\mathrm{M}$ pre-main-sequence stars in two star-forming complexes, TaurusAuriga and Lynds 1641. They found a number of pre-main-sequence stars whose lithium was significantly depleted. They examined the effects of several factors on their estimated abundances, including the possibility that large spotted regions on pre-main-sequence stars enhance the measured lithium abundances. Strom et al. (1989) concluded that at an effective temperature of $5000 \mathrm{~K}$, spots might increase the abundance of lithium in $\mathrm{T}$ Tauri stars by $0.15 \mathrm{dex}$. Thus, they stated that erroneous lithium abundances did not significantly contribute to the observed scatter. Given the uncertainties of their determinations, such a change is not particularly significant. Indeed, a more important source of that scatter is a problem mentioned by those authors. The temperatures of their pre-main-sequence stars, many of which have subgiant and giant luminosities, were assumed from their assigned spectral classes and a temperature calibration for dwarfs. In addition Basri et al. (1991) concluded that the deveiling relation that Strom et al. (1989) used to obtain the corrected equivalent width of the lithium line for their classical $\mathrm{T}$ Tauri stars introduced significant scatter in the results.

In another analysis of pre-main-sequence stars Basri et al. (1991) determined the lithium abundances of $28 \mathrm{~T}$ Tauri stars in the Taurus-Auriga complex. For V410 Tau their observations showed that the lithium line as well as other lines changed with the spot coverage, but they concluded that the observed changes were ambiguous. They noted that the relationship between lithium and spots is more complicated than expected on the basis 
of Giampapa's (1984) prognostication.

Patterer et al. (1993) examined lithium equivalent-width variations of seven weak-lined T Tauri stars including V410 Tau, HDE 283572, Oph 052, and Oph 120S. Despite the moderate uncertainties of their equivalent-width measurements, they concluded that the latter two stars had equivalentwidth variations while variations were likely in three others.

Martín and Claret (1995) discussed the lithium equivalent-width measurements of V410 Tau made by several groups, including Basri et al. (1991) and Patterer et al. (1993). The equivalent widths determined in those individual investigations showed little or no variation with rotational phase, but from a comparison of the average value of each investigation, which ranged from $360 \mathrm{~m} \AA$ to $547 \mathrm{~m} \AA$, they concluded that this star has long-term variations.

Soderblom et al. (1993) determined lithium abundances of over $100 \mathrm{~F}$, $\mathrm{G}$, and K-type Pleiades stars. Despite the presumed coeval nature of the objects, they found a large spread of lithium abundances at a given color for stars of G8 V and later types. In trying to understand that spread, Soderblom et al. (1993) examined the possibility that star spots affected the measured lithium abundances of their stars. They computed some simple spot models in which for a given photospheric temperature they varied the areal coverage of the spots and the spot temperature. Although significant changes in the equivalent width of the lithium line could be produced with large spot regions and spot temperatures that were significantly lower than that of the photosphere, Soderblom et al. (1993) noted such conditions cause additional effects such as color and luminosity changes that were not detected in their sample of stars. When reasonable constraints were placed on the models, little if any increase in the lithium abundance was predicted to result from the spots.

The most extensive investigation of the linkage between lithium and spots was conducted by Pallavicini et al. (1993). While Brown et al. (1989) found that the vast majority of normal giants have abundances that are depleted by a factor of 30 or more relative to the initial Population I $\log$ lithium abundance of about 3.2, the results of recent investigations (Randich et al. 1993, Fekel and Balachandran 1993) showed that some chromospherically active subgiants and giants have significantly larger lithium abundances. In fact, a few post-main-sequence giants have lithium abundances similar to those expected for pre-main-sequence stars! To test the prediction that spot regions could affect the observed lithium line strengths of such stars, Pallavicini et al. (1993) obtained nearly simultaneous photometry and spectroscopy of four well-known extremely active stars, V711 Tau, YY Men, IL Hya, and AB Dor. For those stars they found that their observations had lithium equivalent-width variations of less than $5 \%$. They 
then examined whether their observed limits on such variations were consistent with the presence of cool spots detected by their photometry. The light variations of such stars correspond to the difference between the minimum and maximum spot coverage observed. For photosphere-spot temperature differences greater than $1000 \mathrm{~K}$ the derived spot-coverage factors of their stars were small enough to be consistent with the spectroscopic results. They also computed synthetic spectra for several combinations of photosphere and spot regions, varying the lithium abundance and spot area. Those computations showed that the predicted enhancement factor is most important for very small lithium equivalent widths and decreases rapidly for large lithium equivalent widths.

Finally, Jeffries et al. (1994) obtained high-dispersion blue- and redwavelength spectroscopy and contemporaneous photometry of the star BD $+22^{\circ} 4409$, which they showed to be a young, rapidly rotating, single $\mathrm{K}$ dwarf. The V-band light curve showed an amplitude of about 0.15 mag from which they determined a 10.17 hour rotation period. They measured the lithium equivalent width in 89 spectra obtained during a 7 -hour period. When average equivalent widths were computed from spectra obtained over 45-minute intervals, they found the equivalent width of the blended lithium feature varied by $10 \%$. Unlike the results of Robinson et al. (1986) and Basri et al. (1991), their observed lithium variations were consistent with other data. The lithium line variations appeared to be correlated with equivalent width changes of the nearby $\mathrm{Ca}$ I line, and the time of maximum lithium equivalent width was quite close to the time of maximum starspot coverage, as expected. They estimated the spot coverage needed to produce such lithium equivalent width variations with a spot model that included projection effects and limb darkening. From that comparison Jeffries et al. (1994) concluded that the spot-area coverage derived from the photometric analysis was reasonably consistent with their observed lithium equivalent width variations. Finally, they pointed out that the results of Pallavicini $e t$ al. (1993) could be misleading since they did not include limb darkening.

\section{Prognosis for the patient}

The above results on $\mathrm{T}$ Tauri stars as well as those of Soderblom et al. (1993) and Jeffries et al. (1994), indicate that while under certain conditions significant lithium equivalent-width variations might be observed, in practice the conditions are rarely met. Such conclusions are significantly different from Giampapa's (1984) original prognosis because in reality any actual equivalent-width changes depend on a complex set of factors, including the spot-coverage factor, the temperature difference between the spot and photosphere, the strength of the lithium line, metallicity, and rota- 
tional line broadening, which, if large, can produce significant line blending of lithium with a nearby Fe I line (Pallavicini et al. 1993). Additional simultaneous photometric and high signal-to-noise spectroscopic observations of very active stars such as V410 Tau, II Peg, and HD 12545, which can have large photometric amplitudes, will be important in confirming the results on weak-lined $\mathrm{T}$ Tauri stars and broadening our understanding of the effect of spots on lithium line strength in active stars. Given the conflicting results of some previous studies, measurements should be made of several other temperature sensitive and insensitive lines to confirm the results.

As noted earlier, there is at least one additional parameter involved, beside mass, in the rate of lithium depletion for Population I stars. If star spots are not the primary cause of the observed scatter of abundances, are there other candidates? Soderblom et al. (1993) and García López et al. (1994) have found a connection between high lithium abundance and rapid rotation for Pleiades stars in a certain mass range. While theoretical work on rotating stellar models and lithium depletion has recently begun (e. g. Pinsonneault et al. 1990, Martín and Claret 1995), the results remain preliminary.

For lithium-rich subgiants and giants Fekel and Balachandran (1993) have proposed a scenario in which core angular momentum and possible lithium-rich material are transfered at different rates to the convective outer layers of a post-main-sequence star, when it reaches and begins to ascend the base of the giant branch. The detection of very large lithium abundances in post-main-sequence $\mathrm{K}$ giants such as HR 454, HD 112127, and HR 5409 (Brown et al. 1989, Fekel and Balachandran 1993) means that the identification of an isolated object as a pre-main-sequence star, because the object has a large lithium abundance, may lead to a misdiagnosis. Even more confusion may lie ahead. Skinner et al. (1995) assumed that the "Vega-excess" star HDE 233517 is a very young active K2 dwarf star in part because of its large infrared excess and high galactic latitude. However, despite the dust disk and an apparently undepleted log lithium abundance of 3.3, Fekel et al. (1995) concluded from its radial velocity, galactic latitude, space motion, lack of $\mathrm{H} \alpha$ or strong Ca II emission, and spectral type that it is likely a post-main-sequence $\mathrm{K}$ giant.

\section{References}

Basri G., Martín E. L., Bertout C., 1991, A\&A 252, 625

Boesgaard A. M., 1991, The Formation and Evolution of Star Clusters, ASP Conf. Series 13,463

Brown J. A., Sneden C., Lambert D. L., Dutchover, E., 1989, ApJS 71, 293

Duncan D. K., 1981, ApJ 248, 651

Duncan D. K., Jones, B. F., 1983, ApJ 271, 663

Fekel F. C., Balachandran S., 1993, ApJ 403, 708 
Fekel F. C., et al., 1995, in preparation

García López R. J., Rebolo R., Martín E. L., 1994, A\&A 282, 518

Giampapa M. S., 1984, ApJ 277, 235

Herbig G. H., 1965, ApJ 141, 588

Jeffries R. D., Byrne P. B., Doyle J. G., Anders G. J., James D. J., Lanzafame A. C., 1994, MNRAS 270, 153

Martín E. L., Claret, A., 1995, A\&A, in press

Pallavicini R., Cutispoto G., Randich S., Gratton R., 1993, A\&A 267, 145

Pasquini L., Liu Q., Pallavicini R., 1994, A\&A 287, 191

Patterer R. J., Ramsey L., Huenemoerder D. P., Welty A. D., 1993, AJ 105, 1519

Pilachowski C. A., Sneden C., Hudek D., 1990, AJ 99, 1225

Pinsonneault M. H., Kawaler S. D., Demarque P., 1990, ApJS 74, 501

Randich S., Gratton R., Pallavicini R., 1993, A\&A 273, 194

Robinson R. D., Thompson K., Innes J. L., 1986, Proc. Astron. Soc. Australia 6, 500

Skinner C. J., Sylvester R. J., Graham J. R., Barlow M. J., Meixner M., Keto E., Arens J. F., Jernigan J. G., 1995, ApJ 444, 861

Skumanich A., 1972, ApJ 171, 565

Soderblom D. R., Jones B. F., Balachandran S., Staufer J. R., Duncan D. K., Fedele S. B., Hudon J. D., 1993, AJ 106, 1059

Strom K. M., Wilkin F. P., Strom S. E., Seaman R. L., 1989, AJ 98, 1444

Traub W., Roesler F. L., 1971, ApJ 163, 629 